\title{
A note on 'image' methodology for social work qualitative research: Socially inclusive research methods for service users
}

\begin{abstract}
Sarah Matthews ${ }^{1}$
Abstract: This note offers an analysis of the issues of the social contextual impact of research methodology. Here the author discusses the potential of using 'image based' data collection and analysis methods in social work research and in particular focuses on one possible method, 'rich pictures'. Interest in the use of using image based methods is growing. The author considers the literature which underpins this approach, focussing on the challenges this might bring at all stages of the research process and offers a critique of the ethical and practical dilemmas involved. It will be suggested that such methods have the potential to shift the often criticised power imbalance in all research, including social work research. The author will discuss if this supplementary methodology might increase the ability of service users to participate in research. In this respect, it empathizes with service users who might prefer a non-verbal approach to research inquiry, with more of a range of responsiveness to researchers' question. This note will argue for moving beyond only words in open-ended interviews by social workers to further explore the experiences of service users. As such its use may also be more in accord with the social work values of social justice.
\end{abstract}

Keywords: image based research; rich pictures; non-verbal methodology

1. Staff Tutor, Social Work, The Open University

Address for correspondence: Faculty of Health and Social Care, The Open University, Horlock Building, Walton Hall, Milton Keynes MK76AA.s.a.o.matthews@open.ac.uk

Date of publication (online): 20th September 2012 


\section{Introduction}

Much analysis of the use of image methods for research has until recently concluded that they play, at most, a minor role, or are 'simply a pleasant distraction' (Prosser 1998, p.98). Such non-verbal methods in qualitative research are nonetheless gaining momentum. Here the author discusses the potential of using 'image based' data collection and analysis methods in social work research and in particular focuses on one possible method, 'rich pictures'. This note will argue for moving beyond only words in open-ended interviews by social workers to further explore the experiences of service users. Moreover, do image based methods have the potential to shift the often criticised power imbalance in all research, including social work research, and therefore also be more in accord with the social work values of social justice, as a methodology?

\section{Image based methods}

There is a growing body of literature on the use of image based methods in qualitative research. In 2011 alone, a major text book has been published guiding researchers in 'doing visual research' (Mitchell, 2011), whilst another, recognised text, has published its third edition (Rose, 2011). Other current sources include a handbook (Knowles and Cole 2008), and one part of the Sage Qualitative Research Kit focuses on using visual data in qualitative research (Banks, 2007). These publications reflect the burgeoning interest in the use of image based methods in qualitative research. They detail its roots in the social sciences, including social anthropology and sociology, debate its development and suggest a range of possible methods. So what are these methods and why have they initially been viewed as marginal, and could they be useful to social work researchers?

There are a wide range of methods which come under the umbrella of image based research. The actual medium can include photographs, pieces of material, films, pens and paper. The use of them varies. Some researchers for example might ask participants to seek out already existing personal photographs which might reveal in discussion with the researcher something about the studied topic. Others might ask the particpants to make a film about their experiences which will then be studied by the researcher separately. Particpants can also be asked to create collages and pictures to depict an experience or topic. These might be studied by the researcher separately or form part of the data collection when they are described and interpreted by the particpants to the researcher. The choice of method is governed, as in most qualitative studies, by what is being studied, by the underpinning epistemological proclivity and by practicalities both for the researcher and likely participant. There are in essence three possible relationships said to exist for the protagonists in the 
research data collection method; participants as producers of the data, the researcher and the participant working together to produce the data, or thirdly the researcher both as producer and as interpreter (Mitchell, 2011, p.5).

Motivated by the belief that his own research work would have been enriched if he had drawn on image based methods Prosser, in the late 1990s, undertook a survey of uses at that time. He concluded that image based research methods were viewed as holding limited status. Moreover, he suggested that, in both the manner in which they were used along with the tone by which they were discussed, this lowly status was reinforced (Prosser, 1998, p.98). He contends that images used were either simple line drawings or black and white photographs, the latter being used only to illustrate what the author or subject looked like. He summarised that the debate about the use of image based research methods which viewed their use as ambiguous, open to subjectivity and, in effect, that any analysis of their use raised complex methodological and theoretical issues which further served to weaken them as a research method. This was a view reported to be shared by others (Silverman, 1993). So what are these methodological and theoretical issues which were said to be resulting in the argued marginality of image based research methods?

Echoing the debates about the robustness of qualitative methods in general, the critique of the use of image based methods falls into a number of categories primarily relating to validity. These categories include; authenticity, rigour, trustworthiness, neutrality, objectivity, reliability and transferability to name but a few! There is much discussion in the literature on research methods as to the existence of and reason for attaining quality standards in qualitative research. This dialogue seems now to be more accepting of the opinion that such standards are possible, are necessary and that they should form part of the process of doing the research. Furthermore, that ensuring such quality of research is a responsibility of the researcher. The discussion about the quality of qualitative research has centred on a difference in, and rejection of, terminology and, in turn, accepted benchmarks usually associated with quantitative research where it has hitherto been accepted that ways of ensuring these standards rely upon having set rules and ways of measuring. Such rules are challenged as not being appropriate for ensuring quality in qualitative or interpretivist inquiry (Seale \& Silverman, 1997; Meyrick, 2006; Davies \& Dodd, 2002). In joining the debate about research quality and validity in qualitative research other commentators suggest that hitherto agreed measures for demonstrating robustness are external, objectified, over simplified and mechanical (Koro-Ljungberg, 2010, p.604). The measures KoroLjungberg argues detract from the responsibility of the researcher which he contends should 'take in consideration historical conditions and persisting forms of inequity and oppression while acknowledging the limits of his or her knowledge' (2010, p.604). Whist the fuller detail of the debate about quality and validity in qualitative research is beyond the remit of this article; it is nonetheless interesting to note that if such assertions as to what constitutes robust qualitative research are developed then this too would underpin the claim that all forms of qualitative research, including 
image based research, would be considered robust when researcher responsibility is deemed uppermost in ensuring validity.

Turning to that responsibility, image based research is no different when it comes to the need to address concerns about quality and validity. This is the argument which is being increasingly espoused (Prosser, 1998). The notion that the use of images is more associated with entertainment and persuasion rather than validity is being challenged (Mason, 2005, p.328). There are a number of stances suggested in defence of validity when using image based research methods. For instance; images, when produced by the participant without a given structure from the researcher, can more easily guard against researcher bias. This might otherwise have been present if the researcher had imposed the structure, or directed the research; using participant produced drawings is more likely to accurately represent participant experiences and is therefore reliable and trustworthy. (Kearney \& Hyle, 2004, p.378) Are such stances robust enough? Conversely, such lack of structure and of direction may also mean that the drawings produced might therefore not relate to the research focus, and may therefore be of little use. It is clear that a balance has to be struck. The proposal to use rich pictures as part of my data collection methods, the focus of this article, will present me with a number of challenges, all of which will test my own, and others', belief in the use of rich pictures, and other similar image based methods, as a valid way of collecting and analysing qualitative data. It is to this debate that we now turn.

Despite this disputed background, image based research methods are being considered by researchers in an increasing range of disciplines. What has spurred this increased interest, and why? For some, such methods offer an opportunity to reach levels and forms of knowledge not immediately apprehensible to the researcher through other methods, especially material which may be suppressed and repressed by the conscious mind (Edgar, 1999, p.207). Their use is said to allow the researcher an opportunity to engage with particpants on their level and thereby help to shift what has hitherto been identified as a power imbalance in the researcher-participant relationship Others suggest that 'the use of an integrated approach that involves the use of both visual and word based research methods which offers a way of exploring both the multiplicity and complexity that is the base of much social research interested in human experience' (Guillemin, 2004, p.273). Edgar concludes that, what he refers to as image work, is particularly effective in accessing particpants' implicit awareness (2004, p.209). I am also taken by Mitchell's description of working with the visual which she suggests 'creates a generative space for looking and then looking anew' (2011, p. xiii) Kearney and Hyle comment that the cognitive processes required to draw leads to a more succinct presentation of the key elements of particpants' experiences (2004, p.376) It is the use of image based research methods in other disciplines including social work research that we now turn. 


\section{Image based methods in social work}

There is much less of a body of literature specific to image based research in health and social care. Increasingly however, such methods are beginning to emerge as a way of collecting and analysing data (Guillemin, 2004). One such image based method, as yet untested in social work research, is that of a rich picture. Developed in the early 1980s as part of a Soft Systems Methodology for gathering information (Checkland, 1981), rich pictures were suggested as a way of depicting complex organisational situations using symbols to encapsulate the situation from the viewpoint of the person drawings and to enable communication about their complexity. Their purpose is twofold; to evoke a 'no holds barred' representation of a real situation, including both the factual and subjective information, and, to record this.

There has been no critique undertaken as to the use of rich pictures in social work research. Rather the analysis will need to rely on a critique of similar methods used elsewhere. Here I turn to Edgar who suggests that 'whilst such [image-based] methods might be considered innovative the principles and practice governing the organisation and analysis of data remain within the established qualitative domain' (2004, p.209). A critique of rich pictures should therefore echo a critique of qualitative data collection and analysis generally speaking.

\section{Image based methods and participation}

However, there might be potential of this method and in particular that it might be a way of eliciting hitherto uncharted perspectives about a studied phenomenon with service users less comfortable with speaking about uncomfortable topics. Arguably social work is a profession comfortable with more traditional word based data collection methods. Perhaps a version of rich pictures as a data collection and analysis method would constructively challenge this comfort and in so doing allow particpants, the opportunity of exploring using a new and potentially more participatory perspective. Perhaps this method more accurately reflects social work research and social justice. One commentator suggests that there is richness in visual data which has democratic possibilities (Mitchell, 20011, p.xii). Will using rich pictures enable research particpants to be less dependent and passive? Would the use of a rich picture create a dialogue dimension (Humphries, 2008, p.27)?

Research particpants have knowledge about their situation and the task as researcher as outlined by Humphries is to find ways of making that knowledge available (2008, p.11). Conducting semi-structured interviews with particpants would elicit data and allow an opportunity to analyse and produce findings. Guillemin sees this as an integrated approach (2004, p.273) which provides a succinct presentation (Kearney \& Hyle 2004, p.376). 
The ethics of this methodology are discussed in a recent article, (Clark et al, 2010) which contextualised ethical concerns within existing image based practices and approaches in health and health-related research. Three approaches to research ethics are; consequentionalist, rights-based and principle-based (Clark et al, 2010, p.82). The first involves ethical decisions based on consequences or outcomes of research participation, the second on the rights of individuals including respect for individuals, protection from harm and participatory approaches, the third involves adherence to moral principles including autonomy, non-maleficience, beneficence and justice. Image based research, as other methods, must comply with this context and address such issues. Clark et al (2010) went on to discuss such ethically appropriate issues which impact in all image based research; informed consent, anonymity and confidentiality, and dissemination of visual research. Given the type of research participant, a professional mental health worker might need to determine the extent to which the research subject has the capacity to understand what is being asked of them, whether they will be able to weigh up the consequences and, therefore, be able to make an informed decision about participation.

These issues will apply to both written and image based data. However, will such measures to ensure anonymity for image based data be sufficient? It is usual in written data to allocate anonymous titles to people who might be identified in any transcript and indeed to participants who have provided the data. Is such anonymity possible when using images? The latter will be of particular concern when it comes to dissemination. Will the participant agree to their rich picture being used to illustrate a finding and if so will there be a need to anonymise this in some way and if so, how. If such a method might increase participation disclosure, this may in turn open up the risk that the data can be located and people identified. This is of course a debate whose implications are wider than image based research but is nonetheless an important consideration when justifying this method as a possible participatory one.

Data collection will need to incorporate safeguards not least to allow follow up time, where necessary, and to allow debriefing. Alongside this there should be a clear understanding that the participant can withdraw at any time without prejudice. Ethical issues necessarily involve the acceptance that being part of such a collection method will involve no harm either to themselves, to others or indeed the researcher: the research that, is underpinned by both rights and principle-based approaches. Finally, ethical issues in image based research may also involve legal and moral issues. Clark et al (2010) describe these dilemmas. What they ask is it permissible to record in images? If the image depicts an unlawful or immoral activity how is this to be addressed. In my research there will also be the need to adhere to rules governing professional conduct. Do such considerations therefore compromise the method? Rich pictures evoke no holds barred recording, but within limits! Similar legal complexities apply to ownership. The participant will need to give permission for the image to be used. In both instances the same ethical issues apply as if the 
data collection method was word based only. The consent form will clearly need to address such issues.

The next challenge will be that of convincing the participants. A degree of reluctance is anticipated; initially because of the relative newness and lack of knowledge about the method and, secondly because of an understandable anxiety and perceived embarrassment about being asked to draw. It is hoped that providing information about what a rich picture is and how it will be used as part of the data collection may help ease anxieties which might arise as a result of lack of familiarity with the proposed method. When it comes to the production of a drawing however simplistic or symbolic, Guillemin, in describing her work using drawing as a research method, highlighted the relative paucity of examples of the use of drawings with adults. She wondered if this was because researchers and participants might view such a method as childlike and perhaps only applicable to those less able to articulate using spoken or written words (2004, p.274). For me this raises two responses. The method is proposed in order to elicit data in a new way and not just because particpants may lack a certain ability. It should instead allow them to consider the studied phenomenon from a different perspective. Secondly, the images do not stand alone. For my research the rich pictures will need to be described and interpreted. This is done through the medium of words. For Guillemin this concept of product and process is fundamental (2004, p.274). It is what is referred to elsewhere as the difference between primary texts and production texts (Fiske, 1991, in Mitchell 2011, p.2). The challenge remains as to whether participants will be convinced. For Kearney and Hyle (2004, p.377) 'whether the drawing activity encourages or discourages participation.... may be unpredictable for any given study.' I shall be asking particpants to draw a rich picture as an individual activity but one which they will then share with me as the researcher. Will this be as one commentator describes artistic pleasure (Prosser, 2000), or its opposite, pain, and if the latter will this have negative impact on engagement? If the former, will the participant become so engrossed in doing a good job that what is actually being depicted is not the reality and therefore also a possible threat to validity and rigour.

Having anticipated the possible barriers in data collection which might arise as a consequence of the use of rich pictures to produce data, it now is timely to consider what possible barriers might arise in relation to analysis. This appears relatively straightforward; looking for themes, coding and recoding until no further themes emerge. However what then should happen to the rich pictures themselves. Dissemination of research findings will prove the ultimate test, not least in agreeing whether and how rich pictures can be used to illustrate any findings. Clear rules will have been agreed in relation to anonymity and confidentiality, but will this be as straightforward as simply ascribing a nondescript label? Surely the power of an illustration is just that; that it illustrates! Will attempts to anonymise affect the illustration and in turn the image it is attempting to depict. Finally, to who is the 
illustration ascribed and ultimately will anonymity preclude ownership? For me it is a must that the material is anonymous.

Perhaps there are some research methods which are supplementary and allow social work researchers to find a way of making knowledge available which is both participatory and which reflects the justice ethos of social work as a profession? This it is argued by some should also be the ethical basis of social work research (Humphries, 2008, p.11). It can be argued that in producing an image and being asked to describe the image and what it evokes allows the participant control over the data and participation in its analysis. Others contend that 'the question of who benefits from research and how, is not only questions of ethics but also questions of credibility, trustworthiness and significance of qualitative research' (Koro-Ljungberg, 2010, p604).

\section{Conclusion}

So what of social work research using rich pictures and social justice? Will this particular method convince other social work researchers? Why might the use of rich pictures or, indeed, any similar image based methods, as a data collection tool and subsequent analysis of the data collected, be of interest to them? Will such a method challenge what has been seen as expert driven types of research and therefore possibly sit more easily with social work research and social justice? For those who argue that social work research should reflect social justice, this might well do. For others they may be less convinced. The challenge is to prove that such the use of an image based method integrated with more usual methods of data collection for research proves more useful than a 'pleasant distraction'.

\section{References}

Banks, M. (2007) Using Visual Data in Qualitative Research, London: Sage

Checkland, P., Systems Thinking, Systems Practice London: Wiley

Clark, A., Prosser, J. \& Wiles, R. (2010. Ethical issues in image-based research. Arts \& Health: International Journal for Research, Policy \& Practice, 2, 81-93

Dodd, J. (2002) Qualitative Research and Rigour. Qualitative Health Research, 12, 2, 279-289

Edgar, I.R. 1999) The imagework method in health and social science research. Qualitative Health Research, 9, 198-211

Evans, S., Huxley, P., Webber, M., Katona, C., Gately, C., Mears, A., Medina, J., Pajak, S., and Kendall, T. (2005) The impact of 'statutory duties' on mental health social workers in the UK. Health \& Social Care in the Community, 13, 145-154 
Fiske, J (1991) Understanding Popular Culture Boston MA: Unwin

Guillemin, M. (2004) Understanding illness: Using drawings as a research method. Qualitative Health Research, 14, 272-289

Hotopf, M., Wall, S., Buchanan, A., Wessley, S., and Churchill, R. (2000) Changing patterns in the use of the Mental Health Act 1983 in England, 1984-1996. The British Journal of Psychiatry, 176, 479-484

Humphries, B. (2008) Social Work Research for Social Justice. Basingstoke: Palgrave, Macmillan Kearney, K. S. \& Hyle, A. E. (2004) Drawing out emotions: The use of participant-produced drawings in qualitative inquiry. Qualitative Research, 4, 361-382

Koro-Ljungberg, M., (2010) Validty, Responsibility and aporia. Qualitative Inquiry 16, 603-610

Knowles, J.G.A. and Cole., A.L. (2008) Handbook of the Arts in Qualitative Research. Los Angeles: Sage

Mason, P. (2005) Visual data in applied qualitative research: Lessons from experience. Qualitative research, 5, 325-346

Meyrick, J. (2006 (2010)What is good qualitative research? A comprehensive approach to rigour/quality. Journal of Health Psychology, 11, 5, 799-808

Mitchell, C (2011 (2010)Doing Visual Research, London: Sage

Open University (2005 (2010)T552 systems thinking and practice: Diagramming. Available at: http://systems.open.ac.uk/materials/t522/index.htm [Accessed July 2011]

Prosser, J. (1998) Image-based Research. London: Routledge

Prosser,J. (2000)The moral maze of image ethics. in H. Simons and R. Usher (Eds.), Situated Ethics in Education Research. London: Routledge (pp.116-132)

Quirk, A., Lelliott, P., Audini, B., and Buston, K. (2003) Non-clinical and extra-legal influences on decisions about compulsory admission to psychiatric hospital. Journal of Mental Health, 12,119

Rose, G. (2011)Visual Methodologies. London: Sage

Seale, C. and Silverman, D. (1997) Ensuring rigour in qualitative research. European Journal of Public Health, 7, 379-384

Silverman, D., (1993) The status of image-based research. in J. Prosser, (Ed.) Image-based Research. London: Routledge 